\title{
Vibration of a Euler-Bernoulli uniform beam carrying a rigid body at each end
}

\author{
S. Naguleswaran \\ Department of Mechanical Engineering, University of Canterbury, Christchurch, \\ New Zealand \\ E-Mail: sivapatham.naguleswaran@canterbury.ac.nz
}

\begin{abstract}
Publications on the vibration of a Euler-Bernoulli beam carrying thin discs at the ends are available. In this paper the thin discs are replaced with rigid bodies, whose axial width is included in the analysis. The centre of mass of the bodies is assumed to be on the beam axis but away from the beam end. Among the boundary conditions considered are: the classical clamped, pinned, sliding of free; the lateral translation and rotation of an unrestrained rigid body; the translation and/or rotation of a restrained rigid body, and other special cases. The frequency equations and the first three frequency parameters are tabulated for several sets of the system parameters and selected combinations of 10 boundary conditions.
\end{abstract}

Keywords Euler-Bernoulli beam; vibration; rigid body at each end

\section{Introduction}

The vibration of a cantilever carrying a thin disc is described in the textbook by McCallion [1]. Gorman [2] considered a free-free beam carrying particles at the end and a pinned-pinned beam with thin discs at the ends. Register [3] analysed a beam carrying thin discs at the ends and elastically restrained against rotation and translation. Rama Bhat and Kulkarni [4] and Rama Bhat and Wagner [5] considered a cantilever carrying a heavy body whose centre of mass is offset from the tip. Popplewell and Chang [6] applied the Rayleigh-Ritz method to a cantilever with a heavy body at the tip.

In the present paper, the vibration of a uniform Euler-Bernoulli beam to which a rigid body is attached at each end is analysed using elementary mathematics, mechanics and simple theory of bending. The centre of mass of the rigid body is assumed to be on the beam axis but away from the beam end. This is a valid model in several engineering fields, yet publications are not available. A total of 10 boundary conditions are considered here, which include the classical clamped, pinned, sliding or free, the unrestrained lateral and rotational displacement of a rigid body, the translation and/or rotation of a restrained rigid body, and a rigid body pinned at an arbitrary point and special cases. The principal system parameters are mass, moment of inertia and the centre-of-mass offset. The frequency equations are derived for the combinations of boundary conditions and the first three frequency parameters are tabulated for selected sets of system parameters. Examples are provided to illustrate the calculation of the system parameters. The problems considered in this paper cover a variety of types. The tabulated results may be used to judge frequencies obtained by numerical methods. 


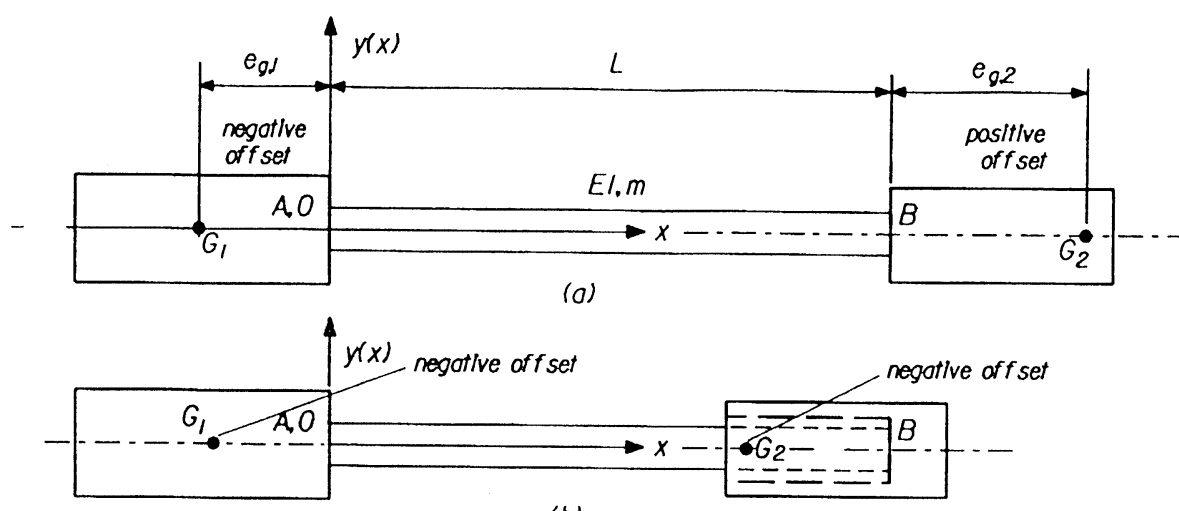

(b)

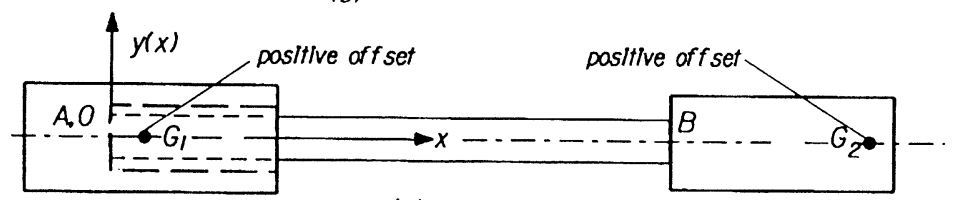

(c)

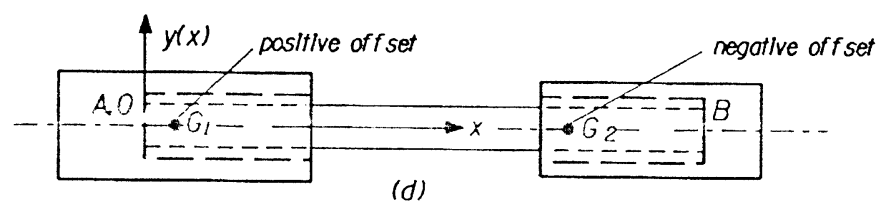

Fig. 1 The beam with the bodies attached: combinations positive or negative centre-ofmass offsets $(a-d)$ and the coordinate system.

\section{Theory}

Fig. 1 shows a Euler-Bernoulli beam, $A B$, of flexural rigidity $E I$, mass per unit length $m$ and length $L$, with two rigid bodies $\left(G_{1}\right.$ and $\left.G_{2}\right)$ attached at $A$ and $B$. The origin, $O$, of the coordinate system shown in Fig. 1(a) coincides with $A$ when the beam is in the undeflected position. The mass of the two rigid bodies are $M_{1}$ and $M_{2}$, and the moments of inertia (about the axis through the centre of mass and normal to the $x-y$ plane) are $J_{1}$ and $J_{2}$, respectively. The centre of mass of $G_{1}$ and $G_{2}$, are assumed to be on the $x$-axis. The centre-of-mass offsets, $A G_{1}=e_{g, 1}$ and $B G_{2}=e_{g, 2}$, are positive in the positive $x$ direction. Some examples of positive/negative combinations of centre-of-mass offsets are shown in Fig. 1(a-d). The vibration analysis is based on the Euler-Bernoulli theory of bending and the sign convention for a positive bending moment and shearing force are shown in Fig. 3(a) (see below).

For free vibration at frequency $\omega$, if $y(x)$ is the amplitude of beam displacement at abscissa $x$, the bending moment, $M(x)$, shearing force, $Q(x)$, and the mode shape differential equation are: 


$$
\begin{aligned}
& M(x)=E I \frac{\mathrm{d}^{2} y(x)}{\mathrm{d} x^{2}} \\
& Q(x)=-E I \frac{\mathrm{d}^{3} y(x)}{\mathrm{d} x^{3}} \\
& E I \frac{\mathrm{d}^{4} y(x)}{\mathrm{d} x^{4}}-m \omega^{2} y(x)=0
\end{aligned}
$$

Next, the dimensionless abscissa $X$, the mode shape $Y(X)$, operators $\mathrm{D}$ and $\mathrm{D}^{n}$, the dimensionless bending moment $M(X)$, the shearing force $Q(X)$, frequency $\Omega$, and the frequency parameter $\alpha$ are defined as follows:

$$
\begin{aligned}
& X=\frac{x}{L} \\
& Y(X)=\frac{y(x)}{L} \\
& \mathrm{D}=\frac{\mathrm{d}}{\mathrm{d} X} \\
& \mathrm{D}^{n}=\frac{\mathrm{d}^{n}}{\mathrm{~d} X^{n}} \\
& M(X)=\frac{M(x) L}{E I} \\
& Q(X)=\frac{Q(x) L^{2}}{E I} \\
& \Omega^{2}=\alpha^{4}=\frac{m \omega^{2} L^{4}}{E I}
\end{aligned}
$$

The expressions in equation 1 can be written in dimensionless form as follows:

$$
\begin{aligned}
& M(X)=\mathrm{D}^{2} Y(X) \\
& Q(X)=-\mathrm{D}^{3} Y(X) \\
& \mathrm{D}^{4} Y(X)-\alpha^{4} Y(X)=0
\end{aligned}
$$

The solution of the dimensionless mode shape differential equation is:

$$
Y(X)=\mathrm{C}_{1} \sin \alpha X+\mathrm{C}_{2} \cos \alpha X+\mathrm{C}_{3} \sinh \alpha X+\mathrm{C}_{4} \cosh \alpha X
$$

where $\mathrm{C}_{1}$ through to $\mathrm{C}_{4}$ are constants of integration.

\section{The boundary conditions}

The boundary conditions considered in this paper are combinations of those shown in Fig. 2. The classical clamped, pinned, sliding and free boundary conditions are shown in Fig. 2( $a_{0}, b_{0}, c_{0}$ and $d_{0}$, respectively). The boundary condition shown in Fig. $2\left(b_{1}\right)$ is as in $\left(b_{0}\right)$ but with a thin disc attached to the beam end; $\left(b_{2}\right)$ is as in $\left(b_{1}\right)$ but with a rigid body; $\left(b_{3}\right)$ is as in $\left(b_{2}\right)$ but pinned at a location on the rigid body; 


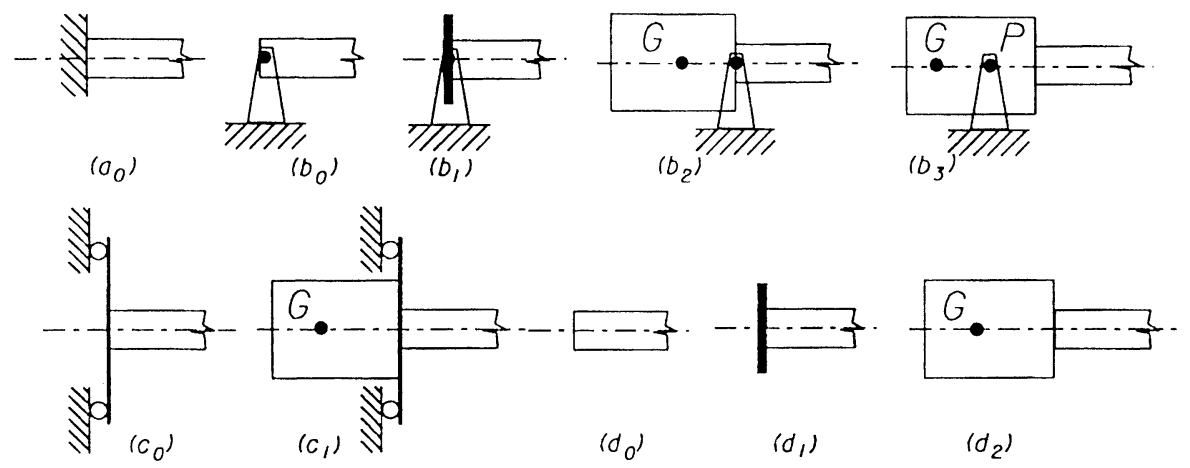

Fig. 2 The 'degenerate' and the 'special' boundary conditions.

$\left(c_{1}\right)$ is as in $\left(c_{0}\right)$ but with the rigid body attached; and $\left(d_{1}\right)$ is as in $\left(d_{0}\right)$ but with a thin disc attached at the end. Finally, the unrestrained rigid body is shown in Fig. $2\left(\mathrm{~d}_{2}\right)$. (These boundary conditions are referred to in Table 3.)

The mass parameter, $\delta_{j}$, the moment of inertia parameter $\Delta_{j}$, the centre-of-mass offset parameter $\varepsilon_{g, j}$ and the pin position parameter $\varepsilon_{p, j}(j=1,2)$ are defined as follows:

$$
\begin{gathered}
\delta_{j}=\frac{M_{j}}{m L} \\
\Delta_{j}=\frac{J_{j}}{m L^{3}} \\
\varepsilon_{g, j}=\frac{e_{g, j}}{L} \\
\varepsilon_{p, j}=\frac{e_{p, j}}{L}
\end{gathered}
$$

in which $j=1$ refers to the end $A$ and $j=2$ to end $B$.

The unrestrained rigid body

The unrestrained rigid body shown in Fig. $2\left(\mathrm{~d}_{2}\right)$ is free to translate and rotate. The free-body diagram of the body at $A\left(\delta_{1}<0, \Delta_{1}<0\right)$ is shown Fig. 3(a) (for positive centre-of-mass offsets). Compatibility of moments and forces leads to the following equations in dimensionless form:

$$
\begin{aligned}
& \mathrm{D}^{2}[Y(0)]+\left[\Delta_{1}+\delta_{1} \varepsilon_{g, 1}^{2}\right] \alpha^{4} \mathrm{D}[Y(0)]+\delta_{1} \varepsilon_{g, 1} \alpha^{4} Y(0)=0 \\
& \mathrm{D}^{3}[Y(0)]-\delta_{1} \varepsilon_{g, 1}^{2} \alpha^{4} \mathrm{D}[Y(0)]-\delta_{1} \alpha^{4} Y(0)=0
\end{aligned}
$$

Equation 4 must satisfy equation 6 , which allows two of the constants in equation 4 to be eliminated and one gets the mode shape as:

$$
Y(X)=\mathrm{A} U(X)+\mathrm{B} V(X)
$$




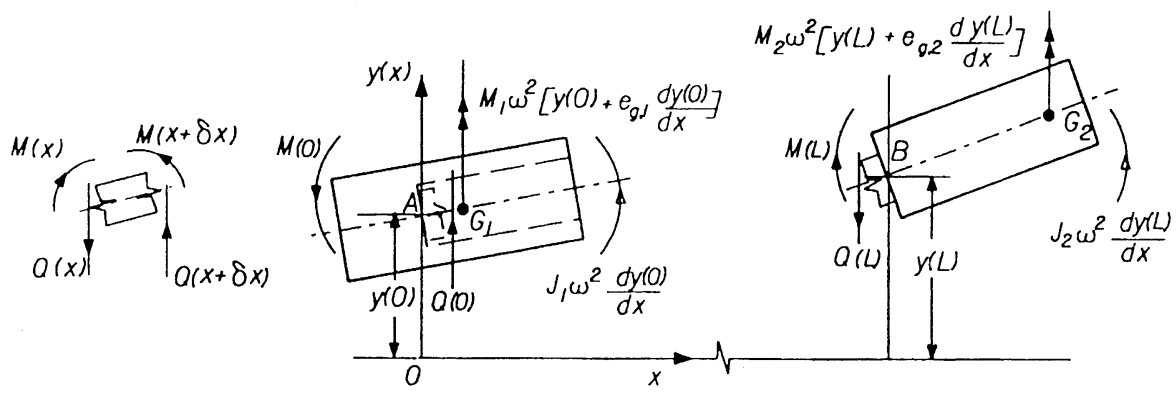

(a)

(b)

(c)

Fig. 3 The sign conventions and the free-body diagrams of the rigid bodies.

in which $\mathrm{A}$ and $\mathrm{B}$ are constants and the mode shape functions $U(X)$ and $V(X)$ are:

$$
\begin{aligned}
& U(X)=\sin \alpha X+g_{1} \cos \alpha X+g_{2} \cosh \alpha X \\
& V(X)=\sinh \alpha X+h_{1} \cos \alpha X+h_{2} \cosh \alpha X
\end{aligned}
$$

where the coefficients $g_{1}, g_{2}, h_{1}$ and $h_{2}$ are:

$$
\begin{aligned}
& g_{1}=\frac{\Delta_{1} \delta_{1} \alpha^{4}-1-2 \delta_{1} \varepsilon_{g, 1} \alpha^{2}}{2 \delta_{1} \alpha} \\
& h_{1}=\frac{\Delta_{1} \delta_{1} \alpha^{4}+1}{2 \delta_{1} \alpha} \\
& g_{2}=-h_{1} \\
& h_{2}=-\frac{\Delta_{1} \delta_{1} \alpha^{4}-1+2 \delta_{1} \varepsilon_{g, 1} \alpha^{2}}{2 \delta_{1} \alpha}
\end{aligned}
$$

An alternative form of the mode shape function is:

$$
\begin{aligned}
& U(X)=\cos \alpha X+g_{1} \sin \alpha X+g_{2} \sinh \alpha X \\
& U(X)=\cosh \alpha X+h_{1} \sin \alpha X+h_{2} \sinh \alpha X
\end{aligned}
$$

in which the coefficients $g_{1}, g_{2}, h_{1}$ and $h_{2}$ are:

$$
\begin{aligned}
& g_{1}=\frac{1-\Delta_{1} \delta_{1} \alpha^{4}-2 \delta_{1} \varepsilon_{g, 1} \alpha^{2}}{2\left(\Delta_{1}+\delta_{1} \varepsilon_{g, 1}^{2}\right) \alpha^{3}} \\
& h_{1}=-\frac{\Delta_{1} \delta_{1} \alpha^{4}+1}{2\left(\Delta_{1}+\delta_{1} \varepsilon_{g, 1}^{2}\right) \alpha^{3}} \\
& g_{2}=-h_{1} \\
& h_{2}=-\frac{1-\Delta_{1} \delta_{1} \alpha^{4}+2 \delta_{1} \varepsilon_{g, 1} \alpha^{2}}{2\left(\Delta_{1}+\delta_{1} \varepsilon_{g, 1}^{2}\right) \alpha^{3}}
\end{aligned}
$$


The 'degenerate' and 'special' boundary conditions

The 'degenerate' boundary conditions occur when $\delta_{1}$ and/or $\Delta_{1}=0$ or $\rightarrow \infty$. 'Special' cases occur when restrictions are imposed on translation or rotation. The mode shape equation ( 8 or 9$)$ are not applicable to these boundary conditions. The mode shape functions $U(X)$ and $V(X)$ for these cases are presented below, in relation to the boundary conditions illustrated in Fig. 2.

Fig. 2( $\left.a_{0}\right)$

This is the classical 'clamped' boundary condition at $A\left(\delta_{1} \rightarrow \infty, \Delta_{1} \rightarrow \infty\right)$, for which $Y(0)=0$ and $\mathrm{D}[Y(0)]=0$. Hence the functions are:

$$
\begin{aligned}
& U(X)=\sin \alpha X-\sinh \alpha X \\
& V(X)=\cos \alpha X-\cosh \alpha X
\end{aligned}
$$

Fig. 2( $\left.b_{0}\right)$

This is the classical 'pinned' boundary condition at $A\left(\delta_{1} \rightarrow \infty, \Delta_{1}=0, \varepsilon_{g, 1}=0\right)$, for which $Y(0)=0$, and $\mathrm{D}^{2}[Y(0)]=0$. Hence the functions are:

$$
\begin{aligned}
& U(X)=\sin \alpha X \\
& V(X)=\sinh \alpha X
\end{aligned}
$$

Fig. 2( $\left.c_{0}\right)$

This is the classical 'sliding' boundary condition at $A\left(\Delta_{1} \rightarrow \infty, \delta_{1}=0\right)$, for which $\mathrm{D}[Y(0)]=0$ and $\mathrm{D}^{3}[Y(0)]=0$. The functions are:

$$
\begin{aligned}
& U(X)=\cos \alpha X \\
& V(X)=\cosh \alpha X
\end{aligned}
$$

Fig. 2(d $)$

This is the classical 'free' boundary condition. Equation ( 8 or 9$)$ is not valid if $\delta_{1}=$ 0 and $\Delta_{1}=0$. Equation 4 must satisfy $\mathrm{D}^{2}[Y(0)]=0, \mathrm{D}^{3}[Y(0)]=0$. The functions $U(X)$ and $V(X)$ are:

$$
\begin{aligned}
& U(X)=\sin \alpha X+\sinh \alpha X \\
& V(X)=\cos \alpha X+\cosh \alpha X
\end{aligned}
$$

Fig. 2( $\left.b_{3}\right)$

The body at $A$ is pinned at $P_{1}$, where $A P_{1}=e_{p, 1}=\varepsilon_{p, 1} L$. The deflection at $P_{1}$ is zero and hence $Y(0)+\varepsilon_{p, 1} \mathrm{D}[Y(0)]=0$. Compatibility of moments at $P_{1}$ leads to $\mathrm{D}^{2}[Y(0)]$ $+\varepsilon_{p, 1} \mathrm{D}^{3}[Y(0)]+\left[\Delta_{1}+\delta_{1}\left(\varepsilon_{g, 1}-\varepsilon_{p, 1}\right)^{2}\right] \alpha^{4} \mathrm{D}[Y(0)]=0$, which must be satisfied by equation 4 and one gets:

$$
\begin{aligned}
& U(X)=\sin \alpha X+g_{1} \cos \alpha X+g_{2} \cosh \alpha X \\
& V(X)=\sinh \alpha X+h_{1} \cos \alpha X+h_{2} \cosh \alpha X
\end{aligned}
$$


in which the coefficients $g_{1}, g_{2}, h_{1}$ and $h_{2}$ are:

$$
\begin{aligned}
& g_{1}=\frac{\left[\Delta_{1}+\delta_{1}\left(\varepsilon_{g, 1}-\varepsilon_{p, 1}\right)^{2}\right] \alpha^{3}-2 \alpha \varepsilon_{p, 1}}{2} \\
& h_{1}=\frac{\left[\Delta_{1}+\delta_{1}\left(\varepsilon_{g, 1}-\varepsilon_{p, 1}\right)^{2}\right] \alpha^{3}}{2} \\
& g_{2}=-h_{1} \\
& h_{2}=-\frac{\left[\Delta_{1}+\delta_{1}\left(\varepsilon_{g, 1}-\varepsilon_{p, 1}\right)^{2}\right] \alpha^{3}+2 \alpha \varepsilon_{p, 1}}{2}
\end{aligned}
$$

Fig. 2( $\left.b_{2}\right)$

In this boundary condition, a body is attached to the beam, which is pinned at $A$. This is a special case of that shown in Fig. $2\left(\mathrm{~b}_{3}\right)$ with $e_{p, 1}=\varepsilon_{p, 1} L=0$. Equation 14 is applicable and the reduced form is:

$$
\begin{aligned}
& U(X)=\sin \alpha X+\frac{\left[\Delta_{1}+\delta_{1} \varepsilon_{g, 1}^{2}\right] \alpha^{3}}{2}(\cos \alpha X-\cosh \alpha X) \\
& V(X)=\sinh \alpha X+\frac{\left[\Delta_{1}+\delta_{1} \varepsilon_{g, 1}^{2}\right] \alpha^{3}}{2}(\cos \alpha X-\cosh \alpha X)
\end{aligned}
$$

Fig. 2( $\left.b_{1}\right)$

Here, a thin disc is attached to the beam, which is pinned at $A$. This is a special case of that shown in Fig. 2(b $\left.\mathrm{b}_{2}\right)$, and equation 15 is applicable, with $\varepsilon_{g, 1}=0$.

Fig. 2( $\left.c_{1}\right)$

A body is attached at $A$ that is constrained to slide transversely without rotation $\left(\Delta_{1} \rightarrow \infty, 0 \leq \delta_{1}<\infty\right)$. One has $\mathrm{D}[Y(0)]=0$ and $\mathrm{D}^{3}[Y(0)]-\delta_{1} \alpha^{4} Y(0)=0$, and the functions are:

$$
\begin{aligned}
& U(X)=\cos \alpha X-\frac{\delta_{1} \alpha(\sin \alpha X-\sinh \alpha X)}{2} \\
& V(X)=\cosh \alpha X-\frac{\delta_{1} \alpha(\sin \alpha X-\sinh \alpha X)}{2}
\end{aligned}
$$

Fig. 2(d $)$

A thin disc is attached at $A$ and the translation and rotation of this disc are unrestrained. This is a special case of that shown in Fig. $2\left(\mathrm{~d}_{2}\right)$. Equation 8 or 9 applies, with $\varepsilon_{g, 1}=0$ subject to $\delta_{1} \neq 0$.

The frequency equations

Consider the unrestrained body at $B$ shown in Fig. $2\left(\mathrm{~d}_{2}\right)$. The free-body diagram (for positive centre-of-mass offset and positive resilient support location) is shown in Fig. 3(b) and one has: 


$$
\begin{aligned}
& \mathrm{D}^{2}[Y(1)]-w_{2,1} \alpha^{4} \mathrm{D}[Y(1)]-w_{2,2} \alpha^{4} Y(1)=0 \\
& \mathrm{D}^{3}[Y(1)]+w_{2,2} \alpha^{4} \mathrm{D}[Y(1)]+w_{2,3} Y(1)=0
\end{aligned}
$$

in which the coefficients $w_{2,1}, w_{2,2}$, and $w_{2,3}$ are:

$$
\begin{aligned}
& w_{2,1}=\left(\Delta_{1}+\delta_{2} \delta_{g, 2}^{2}\right) \alpha^{4} \\
& w_{2,2}=\delta_{2} \varepsilon_{g, 2} \alpha^{4} \\
& w_{2.3}=\delta_{2} \alpha^{4}
\end{aligned}
$$

The mode shape equation 7 must satisfy equation 17 . For a non-trivial solution, the coefficient matrix must be singular. For the unrestrained body at $B$, the frequency equation is:

$$
\begin{aligned}
& \left\{\mathrm{D}^{2}[U(1)]-w_{2,1} \mathrm{D}[U(1)]-w_{2,2} U(1)\right\}\left\{\mathrm{D}^{3}[V(1)]+w_{2,2} \mathrm{D}[V(1)]+w_{3,2} V(1)\right\} \\
& \left\{\mathrm{D}^{3}[U(1)]+w_{2,2} \mathrm{D}[U(1)]+w_{3,2} U(1)\right\}\left\{\mathrm{D}^{2}[V(1)]-w_{2,1} \mathrm{D}[V(1)]-w_{2,2} V(1)\right\}=0
\end{aligned}
$$

in which $U(1), V(1), \mathrm{D}[U(1)], \mathrm{D}[V(1)]$ etc. are obtained from the functions applicable to the boundary conditions at $A$.

For the degenerate and 'special' types of support (boundary condition) at $B$, the frequency equations are as set out below.

Fig. 2( $\left.a_{0}\right)$

This is the classical 'clamped' boundary condition at $B\left(\delta_{2} \rightarrow \infty, \Delta_{2} \rightarrow \infty\right)$. Equation 7 must satisfy $Y(1)=0, \mathrm{D}[Y(1)]=0$. The frequency equation is:

$$
U(1) \mathrm{D}[V(1)]-\mathrm{D}[U(1)] V(1)=0
$$

Fig. 2( $\left.b_{0}\right)$

This is the classical 'pinned' boundary condition at $B\left(\delta_{2} \rightarrow \infty, \Delta_{2}=0, \varepsilon_{g, 2}=0\right)$. Equation 7 must satisfy $Y(1)=0, \mathrm{D}^{2}[Y(1)]=0$. The frequency equation is:

$$
U(1) \mathrm{D}^{2}[V(1)]-\mathrm{D}^{2}[U(1)] V(1)=0
$$

Fig. 2( $\left.c_{0}\right)$

This is the classical 'sliding' boundary condition at $B\left(\Delta_{2} \rightarrow \infty, \delta_{2}=0\right)$. Equation 7 must satisfy $Y(1)=0, \mathrm{D}^{3} Y(1)=0$, and the frequency equation is:

$$
\mathrm{D}[U(1)] \mathrm{D}^{3}[V(1)]-\mathrm{D}^{3}[U(1)] \mathrm{D}[V(1)]=0
$$

Fig. 2(d $)$

This is the classical 'free' boundary condition at $B$. One has $\mathrm{D}^{2} Y(1)=0, \mathrm{D}^{3} Y(1)=0$ and the frequency equation is:

$$
\mathrm{D}^{2}[U(1)] \mathrm{D}^{3}[V(1)]-D^{3}[\mathrm{U}(1)] \mathrm{D}^{2}[V(1)]=0
$$

Fig. 2( $\left.b_{3}\right)$

Here, the body at $B$ is pinned at $P_{2}$, where $B P_{2}=e_{p, 2}=\varepsilon_{p, 2} L$. Equation 7 must satisfy $Y(1)+\varepsilon_{p, 2} \mathrm{D}[Y(1)]=0, \mathrm{D}^{2}[Y(1)]+\varepsilon_{p, 2} \mathrm{D}^{3}[Y(1)]-w_{2}^{*} \mathrm{D}[Y(1)]=0$, where the coefficient $w_{2}^{*}=\left[\Delta_{2}+\delta_{2}\left(\varepsilon_{g, 2}-\varepsilon_{p, 2}\right)^{2}\right] \alpha^{4}$. The frequency equation is: 


$$
\begin{aligned}
& \left\{U(1)+\varepsilon_{p, 2} \mathrm{D}[U(1)]\right\}\left\{\mathrm{D}^{2}[V(1)]+\varepsilon_{p, 2} \mathrm{D}^{3}[V(1)]-w_{2}^{*} \mathrm{D}[V(1)]\right\} \\
& \quad-\left\{\mathrm{D}^{2}[U(1)]+\varepsilon_{p, 2} \mathrm{D}^{3}[U(1)]-w_{2}^{*} \mathrm{D}[U(1)]\right\}\left\{V(1)+\varepsilon_{p, 2} \mathrm{D}[V(1)]\right\}=0
\end{aligned}
$$

Fig. 2( $\left.b_{2}\right)$

The body is attached at $B$ and pinned at $B$. The frequency equation (23) is applicable, with $\varepsilon_{p, 2}=0$. The reduced form is:

$$
\begin{aligned}
& U(1)\left\{\mathrm{D}^{2}[V(1)]-\left[\Delta_{2}+\delta_{2} \varepsilon_{g, 2}^{2}\right] \alpha^{4} \mathrm{D}[V(1)]\right\} \\
& -\left\{\mathrm{D}^{2}[U(1)]-\left[\Delta_{2}+\delta_{2} \varepsilon_{g, 2}^{2}\right] \alpha^{4} \mathrm{D}[U(1)]\right\} U(1)=0
\end{aligned}
$$

Fig. 2( $\left.b_{1}\right)$

A thin disc is attached at $B$ and pinned at $B$. The frequency equation (23) is applicable, with $\varepsilon_{p, 2}=0$ and $\varepsilon_{g, 2}=0$. The reduced form is:

$$
U(1)\left\{\mathrm{D}^{2}[V(1)]-\Delta_{2} \alpha^{4} \mathrm{D}[V(1)]\right\}-\left\{\mathrm{D}^{2}[U(1)]-\Delta_{2} \alpha^{4} \mathrm{D}[U(1)]\right\} V(1)=0
$$

Fig. 2( $\left.c_{1}\right)$

The body is attached at $B$ but is constrained to slide transversely without rotation $\left(\Delta_{2} \rightarrow \infty, 0 \leq \delta_{2}<\infty\right)$. Equation 7 must satisfy $\mathrm{D}[Y(1)]=0$ and $\mathrm{D}^{3}[Y(1)]+\delta_{2} \alpha^{4} Y(1)$ $=0$. The frequency equation is:

$$
\mathrm{D}[U(1)]\left\{\mathrm{D}^{3}[V(1)]+\delta_{2} \alpha^{4} V(1)\right\}-\left\{\mathrm{D}^{3}[U(1)]+\delta_{2} \alpha^{4} U(1)\right\} \mathrm{D}[V(1)]=0
$$

Fig. 2(d $)$

A thin disc is attached at $B$ but lateral translation and rotation are unrestrained. The frequency equation (17) is applicable, with $\varepsilon_{g, 2}=0$. The reduced form is:

$$
\begin{aligned}
& \left\{\mathrm{D}^{2}[U(1)]-\Delta_{2} \alpha^{4} \mathrm{D}[U(1)]\right\}\left\{\mathrm{D}^{3}[V(1)]+\delta_{2} \alpha^{4} V(1)\right\} \\
& -\left\{\mathrm{D}^{3}[U(1)]+\delta_{2} \alpha^{4} U(1)\right\}\left\{\mathrm{D}^{2}[V(1)]-\Delta_{2} \alpha^{4} \mathrm{D}[V(1)]\right\}=0
\end{aligned}
$$

\section{The natural frequency calculations}

The system parameters are: $\delta_{1}, \Delta_{1}, \varepsilon_{g, 1}, \varepsilon_{p, 1}, \delta_{2}, \Delta_{2}, \varepsilon_{g, 2}, \varepsilon_{p, 2}$. Depending on the boundary conditions, some of the parameters will be zero or will not enter the frequency equation. As there are eight parameters, it is not possible here to present all the results in graphical or tabular form.

The end-bodies unrestrained

The boundary condition (at each end) is that shown in Fig. $2\left(\mathrm{~d}_{2}\right)$ and is denoted by $\left(\mathrm{d}_{2}, \mathrm{~d}_{2}\right)$. Note that $\varepsilon_{p, 1}$ and $\varepsilon_{p, 2}$ are not needed here. For the selected set of system parameters $\delta_{1}, \Delta_{1}, \varepsilon_{g, 1}, \delta_{2}, \Delta_{2}, \varepsilon_{g, 2}$ (none of which is infinity), a trial frequency parameter $\alpha$ (of 0.1 , say) was assumed. The coefficients $g_{1}, g_{2}, h_{1}$, and $h_{2}$ were calculated from equation $8 \mathrm{a}$ and inserted into equation 8 to obtain $U(1), V(1)$ and hence $\mathrm{D}[U(1)], \mathrm{D}[V(1)]$ etc. by straightforward differentiation of $U(X)$ and $V(X)$. From equation $17 \mathrm{a}, w_{2,1}, w_{2,2}$, and $w_{2,3}$ were calculated and one is now in a position to 
TABLE 1 The first three frequency parameters for a beam carrying unrestrained rigid bodies at its ends

\begin{tabular}{|c|c|c|c|c|c|c|c|c|c|c|}
\hline \multirow[b]{2}{*}{$\left(\delta_{1}, \Delta_{1}\right)$} & \multirow[b]{2}{*}{$\varepsilon_{g, 1}$} & \multicolumn{3}{|c|}{$\varepsilon_{g, 2}=-0.3$} & \multicolumn{3}{|c|}{$\varepsilon_{g, 2}=0.0$} & \multicolumn{3}{|c|}{$\varepsilon_{g, 2}=0.3$} \\
\hline & & $\alpha_{1}$ & $\alpha_{2}$ & $\alpha_{3}$ & $\alpha_{1}$ & $\alpha_{2}$ & $\alpha_{3}$ & $\alpha_{1}$ & $\alpha_{2}$ & $\alpha_{3}$ \\
\hline \multirow[t]{3}{*}{$(0.1,0.2)$} & -0.8 & 1.5484 & 3.0192 & 5.8155 & 1.5552 & 3.0502 & 5.8252 & 1.5490 & 3.0875 & 5.8507 \\
\hline & 0.0 & 1.6307 & 2.9460 & 5.7347 & 1.6372 & 2.9775 & 5.7448 & 1.6324 & 3.0149 & 5.7706 \\
\hline & 0.4 & 1.6372 & 2.8948 & 5.7172 & 1.6413 & 2.9277 & 5.7274 & 1.6349 & 2.9662 & 5.7535 \\
\hline \multirow[t]{3}{*}{$(5.0,1.0)$} & -0.8 & 1.0435 & 2.1104 & 4.9684 & 1.0851 & 2.1636 & 4.9737 & 1.0930 & 2.2547 & 5.0041 \\
\hline & 0.0 & 1.1291 & 1.6862 & 4.8164 & 1.1580 & 1.7757 & 4.8228 & 1.1487 & 1.9123 & 4.8553 \\
\hline & 0.4 & 1.1562 & 1.5055 & 4.8133 & 1.1271 & 1.6450 & 4.8197 & 1.0941 & 1.8131 & 4.8526 \\
\hline
\end{tabular}

System parameters: $\delta_{1}, \Delta_{1}, \varepsilon_{g, 1}$ and $\varepsilon_{g, 2}$ (in table) and $\delta_{2}=\delta_{1}+0.1, \Delta_{2}=\Delta_{1}+0.2$.

calculate the left-hand side of equation 18 to yield a 'remainder'. The trial value of the frequency parameter was changed in steps of 0.1 and calculations were repeated until a sign change in the 'remainder' occurred. This indicates a 'range' within which a root is present. The 'search' was made in this 'range' with a step change of 0.01 in the trial frequency, thus narrowing the 'range' within which a root was present. An iteration procedure based on linear interpolation was invoked to obtain the root to a pre-assigned accuracy. The 'search' was continued from this root to obtain the second root and so on.

The first three non-zero frequency parameters are tabulated in Table 1 for various combinations of $\delta_{1}=0.1$ or $5.0, \Delta_{1}=0.2$ or $1.0, \varepsilon_{g, 1}=-0.8$ or 0.0 or $0.4, \varepsilon_{g, 2}=-0.3$ or 0.0 or 0.3 . For convenience the right-hand body was chosen so that $\delta_{2}=\delta_{1}+0.1$, $\Delta_{2}=\Delta_{1}+0.2$. An increase in the mass and/or moment of intertia of the rigid bodies results in a decrease in the frequency - the effect of a change in $\Delta$ being greater than that of $\delta$. The effect of a change in $\varepsilon_{g, 1}$ or $\varepsilon_{g, 2}$ on the frequency is smaller. For thin discs at the ends, $\varepsilon_{g, 1}=\varepsilon_{g, 2}=0$.

\section{The rigid bodies pinned}

This is the boundary condition $\left(\mathrm{b}_{3}, \mathrm{~b}_{3}\right)$. The pin location parameters $\varepsilon_{p, 1}, \varepsilon_{p, 2}$ are needed here. For example calculations, the parameters chosen were $\delta_{1}=0.5, \Delta_{1}=$ $0.1, \delta_{2}=1.0, \Delta_{2}=0.2$ and combinations were used of $\varepsilon_{g, 1}=-0.1$ or $0.1, \varepsilon_{p, 1}=-0.3$ or $0.3, \varepsilon_{g, 2}=-0.3,0.0$ or $0.3, \varepsilon_{p, 2}=-0.4,0.0$ or 0.4 . The functions $U(X)$ and $V(X)$ were obtained from equation 14 and the frequency equation is 23 . The first three frequency parameters are tabulated in Table 2.

\section{The 'degenerate' cases}

The relevant system parameters were chosen from the set $\delta_{1}=0.1, \Delta_{1}=0.2, \varepsilon_{g, 1}=$ $-0.1, \varepsilon_{p, 1}=-0.3, \delta_{2}=0.2, \Delta_{2}=0.3, \varepsilon_{g, 2}=0.3, \varepsilon_{p, 2}=0.2$. For example, if the end body at $A$ is supported as in Fig. 2(c $\left.c_{1}\right), \Delta_{1}, \varepsilon_{g, 1}$ and $\varepsilon_{p, 1}$ will not enter the calculations; or if the end body $B$ is supported as in Fig. $2\left(b_{3}\right), \delta_{2}, \Delta_{2}, \varepsilon_{g, 2}$ and $\varepsilon_{p, 2}$ will enter the calculations. 
TABLE 2 The first three frequency parameters for a beam with pinned rigid bodies at its ends

\begin{tabular}{|c|c|c|c|c|c|c|c|c|c|c|}
\hline \multirow[b]{2}{*}{$\varepsilon_{g, 1}$} & \multirow[b]{2}{*}{$\varepsilon_{p, 1}, \varepsilon_{p, 2}$} & \multicolumn{3}{|c|}{$\varepsilon_{g, 2}=-0.3$} & \multicolumn{3}{|c|}{$\varepsilon_{g, 2}=0.0$} & \multicolumn{3}{|c|}{$\varepsilon_{g, 2}=0.3$} \\
\hline & & $\alpha_{1}$ & $\alpha_{2}$ & $\alpha_{3}$ & $\alpha_{1}$ & $\alpha_{2}$ & $\alpha_{3}$ & $\alpha_{1}$ & $\alpha_{2}$ & $\alpha_{3}$ \\
\hline \multirow[t]{3}{*}{-0.1} & $(-0.3,-0.4)$ & 1.47386 & 2.5730 & 5.1106 & 1.30403 & 2.5718 & 5.0988 & 1.11783 & 2.5711 & 5.0903 \\
\hline & $(-0.3,0.0)$ & 1.59533 & 2.6983 & 5.1044 & 1.70818 & 2.7580 & 5.1153 & 1.59533 & 2.6983 & 5.1044 \\
\hline & $(-0.3,0.4)$ & 1.39562 & 2.7671 & 5.1649 & 1.52698 & 2.8973 & 5.2334 & 1.60990 & 3.0334 & 5.3219 \\
\hline \multirow[t]{3}{*}{0.1} & $(0.3,-0.4)$ & 1.26950 & 1.9107 & 4.7721 & 1.14345 & 1.8767 & 4.7601 & 0.99186 & 1.8545 & 4.7515 \\
\hline & $(0.3,0.0)$ & 1.81808 & 1.9221 & 4.7745 & 1.82568 & 2.0925 & 4.7896 & 1.81808 & 1.9221 & 4.7745 \\
\hline & $(0.3,0.4)$ & 1.71845 & 1.9926 & 4.8461 & 1.76603 & 2.2118 & 4.9291 & 1.77866 & 2.4123 & 5.0338 \\
\hline
\end{tabular}

System parameters: $\varepsilon_{g, 1} \varepsilon_{p, 1}, \delta_{2}, \Delta_{2}, \varepsilon_{g, 2}, \varepsilon_{p, 2}$ (in table) and $\delta_{1}=0.5, \Delta_{1}=0.1, \delta_{2}=1.0, \Delta_{2}=0.2$.

Consider, for example, the boundary conditions $\left(\mathrm{c}_{1}, \mathrm{~b}_{2}\right)$, that is, the left-hand endbody system $(A)$ is sliding and the right-hand end-body system is pinned at $B$. For a trial frequency parameter, $U(1), V(1), \mathrm{D}[U(1)], \mathrm{D}[V(1)]$ etc. were calculated from equation 16 and inserted into the left-hand side of equation 24 with $\varepsilon_{p, 2}=0$ to obtain the 'remainder' of the frequency equation and the procedure described earlier was used to determine the roots. The first three frequency parameters were calculated for 100 combinations of boundary conditions $a_{0}-d_{2}$. Combinations of the classical 'clamped', 'pinned', 'sliding' or 'free' supports, for example $\left(\mathrm{a}_{0}, \mathrm{~d}_{0}\right)$, were deleted and the rest are tabulated in Table 3.

\section{Conjoint systems}

If one denotes the frequency parameter $\alpha\left[(i, j),\left(\delta_{1}, \Delta_{1}, \varepsilon_{g, 1}, \varepsilon_{p, 1}\right),\left(\delta_{2}, \Delta_{2}, \varepsilon_{g, 2}, \varepsilon_{p, 2}\right)\right]$ in which $i$ or $j \equiv \mathrm{a}_{0}$ through to $\mathrm{d}_{2}$ denotes the boundary condition at $A$ and $B$, respectively, then:

$$
\begin{aligned}
& \alpha\left[(i, j),\left(\delta_{1}=\mathrm{d}_{1}, \Delta_{1}=\mathrm{D}_{1}, \varepsilon_{g, 1}=e_{1}, \varepsilon_{p, 1}=p_{1}\right),\right. \\
& \left.\quad\left(\delta_{2}=\mathrm{d}_{2}, \Delta_{2}=\mathrm{D}_{2}, \varepsilon_{g, 2}=e_{2}, \varepsilon_{p, 2}=p_{2}\right)\right] \\
& \quad=\alpha\left[(j, i),\left(\delta_{1}=\mathrm{d}_{2}, \Delta_{1}=\mathrm{D}_{2}, \varepsilon_{g, 1}=-e_{2}, \varepsilon_{p, 1}=-p_{2}\right),\right. \\
& \left.\quad\left(\delta_{2}=\mathrm{d}_{1}, \Delta_{2}=\mathrm{D}_{1}, \varepsilon_{g, 2}=-e_{1}, \varepsilon_{p, 2}=-p_{1}\right)\right]
\end{aligned}
$$

The frequency parameters of the corresponding conjoint systems are presented in Table 4.

\section{Limiting cases}

If $\delta$ is increased indefinitely and $\Delta$ is kept small, a pseudo-pinned condition is approached asymptotically. A near-sliding condition is approached if $\Delta$ is increased indefinitely and $\delta$ is kept small. If $\delta$ and $\Delta$ are increased indefinitely, a pseudoclamped condition is approached. In Table 4 the first three frequency parameters of $\left(\mathrm{d}_{2}, \mathrm{~d}_{2}\right)$ systems are tabulated for three combinations of $\varepsilon_{g, 1}$ and $\varepsilon_{g, 2}$ and selected combinations of $\delta_{1}=1,10,10^{2}, 10^{3}, 10^{4}, \Delta_{1}=1,10^{2}, 10^{3}$. For convenience, $\delta_{1}=\delta_{2}$ and 
TABLE 3 The first three non-zero frequency parameters for 'degenerate' cases

\begin{tabular}{|c|c|c|c|c|c|c|c|}
\hline $\mathrm{BC}$ & $\alpha_{1}$ & $\alpha_{2}$ & $\alpha_{3}$ & $\mathrm{BC}$ & $\alpha_{1}$ & $\alpha_{2}$ & $\alpha_{3}$ \\
\hline$\left(a_{0}, b_{1}\right)$ & 1.89568 & 4.76187 & 7.86012 & $\left(\mathrm{~d}_{0}, \mathrm{~b}_{1}\right)$ & 1.22555 & 2.59555 & 5.51804 \\
\hline$\left(a_{0}, b_{2}\right)$ & 1.86912 & 4.76005 & 7.85972 & $\left(\mathrm{~d}_{0}, \mathrm{~b}_{2}\right)$ & 1.21368 & 2.58370 & 5.51689 \\
\hline$\left(a_{0}, b_{3}\right)$ & 2.10774 & 4.84091 & 7.89750 & $\left(\mathrm{~d}_{0}, \mathrm{~b}_{3}\right)$ & 1.12535 & 2.76233 & 5.58246 \\
\hline$\left(\mathrm{a}_{0}, \mathrm{c}_{1}\right)$ & 2.13339 & 5.17434 & 8.21537 & $\left(\mathrm{~d}_{0}, \mathrm{c}_{1}\right)$ & 2.91963 & 5.92693 & 8.98279 \\
\hline$\left(a_{0}, d_{1}\right)$ & 1.24777 & 2.29964 & 5.19317 & $\left(\mathrm{~d}_{0}, \mathrm{~d}_{1}\right)$ & 1.30736 & 3.00884 & 5.93978 \\
\hline$\left(\mathrm{a}_{0}, \mathrm{~d}_{2}\right)$ & 1.19272 & 2.36083 & 5.23072 & $\left(\mathrm{~d}_{0}, \mathrm{~d}_{2}\right)$ & 1.26423 & 3.06090 & 5.97410 \\
\hline$\left(b_{0}, b_{1}\right)$ & 1.75017 & 3.98227 & 7.07807 & $\left(c_{1}, a_{0}\right)$ & 2.23492 & 5.29438 & 8.35270 \\
\hline$\left(b_{0}, b_{2}\right)$ & 1.72642 & 3.97909 & 7.07753 & $\left(c_{1}, b_{0}\right)$ & 1.50065 & 4.52977 & 7.58563 \\
\hline$\left(b_{0}, b_{3}\right)$ & 1.83923 & 4.08803 & 7.12191 & $\left(c_{1}, b_{1}\right)$ & 1.20618 & 2.49072 & 5.31641 \\
\hline$\left(b_{0}, c_{1}\right)$ & 1.44363 & 4.41515 & 7.45046 & $\left(c_{1}, b_{2}\right)$ & 1.19529 & 2.47779 & 5.31515 \\
\hline$\left(b_{0}, d_{1}\right)$ & 1.84907 & 4.44420 & 7.45723 & $\left(c_{1}, b_{3}\right)$ & 1.10208 & 2.66062 & 5.38252 \\
\hline$\left(b_{0}, d_{2}\right)$ & 1.87873 & 4.48660 & 7.48602 & $\left(c_{1}, c_{0}\right)$ & 3.01154 & 6.05485 & 9.12124 \\
\hline$\left(b_{1}, a_{0}\right)$ & 2.08949 & 4.77801 & 7.86359 & $\left(c_{1}, c_{1}\right)$ & 2.79818 & 5.71658 & 8.69513 \\
\hline$\left(b_{1}, b_{0}\right)$ & 1.92148 & 4.01044 & 7.08285 & $\left(c_{1}, d_{0}\right)$ & 2.29117 & 5.28872 & 8.35307 \\
\hline$\left(b_{1}, b_{1}\right)$ & 1.64768 & 2.23821 & 4.80797 & $\left(c_{1}, d_{1}\right)$ & 1.30259 & 2.89330 & 5.73041 \\
\hline$\left(b_{1}, b_{2}\right)$ & 1.63212 & 2.22783 & 4.80625 & $\left(c_{1}, d_{2}\right)$ & 1.25825 & 2.94502 & 5.76468 \\
\hline$\left(b_{1}, b_{3}\right)$ & 1.63882 & 2.39541 & 4.88370 & $\left(\mathrm{~d}_{0}, \mathrm{~b}_{1}\right)$ & 2.24177 & 4.72669 & 7.86167 \\
\hline$\left(b_{1}, c_{0}\right)$ & 1.30353 & 2.69435 & 5.52829 & $\left(\mathrm{~d}_{0}, \mathrm{~b}_{2}\right)$ & 2.22592 & 4.72483 & 7.86127 \\
\hline$\left(b_{1}, c_{1}\right)$ & 1.25190 & 2.52689 & 5.20963 & $\left(\mathrm{~d}_{0}, \mathrm{~b}_{3}\right)$ & 2.35734 & 4.81044 & 7.89896 \\
\hline$\left(b_{1}, d_{0}\right)$ & 2.36678 & 4.74325 & 7.86514 & $\left(\mathrm{~d}_{0}, \mathrm{c}_{1}\right)$ & 2.23704 & 5.16435 & 8.21598 \\
\hline$\left(b_{1}, d_{1}\right)$ & 1.64975 & 2.60072 & 5.22774 & $\left(\mathrm{~d}_{0}, \mathrm{~d}_{1}\right)$ & 2.42940 & 5.18308 & 8.22112 \\
\hline$\left(b_{1}, d_{2}\right)$ & 1.64353 & 2.64397 & 5.26436 & $\left(\mathrm{~d}_{0}, \mathrm{~d}_{2}\right)$ & 2.46991 & 5.22150 & 8.24777 \\
\hline$\left(b_{2}, a_{0}\right)$ & 2.08701 & 4.77776 & 7.86354 & $\left(\mathrm{~d}_{1}, \mathrm{a}_{0}\right)$ & 1.36686 & 2.43137 & 5.31760 \\
\hline$\left(b_{2}, b_{0}\right)$ & 1.91931 & 4.01002 & 7.08278 & $\left(\mathrm{~d}_{1}, \mathrm{~b}_{0}\right)$ & 1.98292 & 4.56536 & 7.59411 \\
\hline$\left(b_{2}, b_{1}\right)$ & 1.64706 & 2.23638 & 4.80773 & $\left(\mathrm{~d}_{1}, \mathrm{~b}_{1}\right)$ & 1.68390 & 2.60791 & 5.33908 \\
\hline$\left(b_{2}, b_{2}\right)$ & 1.63155 & 2.22595 & 4.80602 & $\left(\mathrm{~d}_{1}, \mathrm{~b}_{2}\right)$ & 1.67434 & 2.59800 & 5.33786 \\
\hline$\left(b_{2}, b_{3}\right)$ & 1.63786 & 2.39395 & 4.88348 & $\left(\mathrm{~d}_{1}, \mathrm{~b}_{3}\right)$ & 1.66001 & 2.76127 & 5.40399 \\
\hline$\left(\mathrm{b}_{2}, \mathrm{c}_{0}\right)$ & 1.30263 & 2.69296 & 5.52814 & $\left(\mathrm{~d}_{1}, \mathrm{c}_{0}\right)$ & 1.43787 & 3.12087 & 6.07072 \\
\hline$\left(b_{2}, c_{1}\right)$ & 1.25118 & 2.52530 & 5.20946 & $\left(\mathrm{~d}_{1}, \mathrm{c}_{1}\right)$ & 1.42654 & 2.92083 & 5.73462 \\
\hline$\left(b_{2}, d_{0}\right)$ & 2.36508 & 4.74300 & 7.86509 & $\left(\mathrm{~d}_{1}, \mathrm{~d}_{0}\right)$ & 2.53420 & 5.31167 & 8.35955 \\
\hline$\left(b_{2}, d_{1}\right)$ & 1.64903 & 2.59935 & 5.22757 & $\left(\mathrm{~d}_{1}, \mathrm{~d}_{1}\right)$ & 1.68591 & 2.99639 & 5.74822 \\
\hline$\left(b_{2}, d_{2}\right)$ & 1.64271 & 2.64267 & 5.26419 & $\left(\mathrm{~d}_{1}, \mathrm{~d}_{2}\right)$ & 1.67441 & 3.04338 & 5.78210 \\
\hline$\left(b_{3}, a_{0}\right)$ & 2.35646 & 4.95836 & 7.95961 & $\left(\mathrm{~d}_{2}, \mathrm{a}_{0}\right)$ & 1.35325 & 2.44616 & 5.32641 \\
\hline$\left(b_{3}, b_{0}\right)$ & 1.98902 & 4.23635 & 7.19324 & $\left(\mathrm{~d}_{2}, \mathrm{~b}_{0}\right)$ & 1.98964 & 4.57549 & 7.60045 \\
\hline$\left(b_{3}, b_{1}\right)$ & 1.56057 & 2.50654 & 4.98393 & $\left(\mathrm{~d}_{2}, \mathrm{~b}_{1}\right)$ & 1.67890 & 2.62003 & 5.34775 \\
\hline$\left(b_{3}, b_{2}\right)$ & 1.54404 & 2.49765 & 4.98247 & $\left(\mathrm{~d}_{2}, \mathrm{~b}_{2}\right)$ & 1.66906 & 2.61027 & 5.34653 \\
\hline$\left(b_{3}, b_{3}\right)$ & 1.54473 & 2.64895 & 5.05283 & $\left(\mathrm{~d}_{2}, \mathrm{~b}_{3}\right)$ & 1.65534 & 2.77253 & 5.41241 \\
\hline$\left(b_{3}, c_{0}\right)$ & 1.11520 & 2.96721 & 5.68171 & $\left(\mathrm{~d}_{2}, \mathrm{c}_{0}\right)$ & 1.42710 & 3.13392 & 6.07856 \\
\hline$\left(b_{3}, c_{1}\right)$ & 1.06157 & 2.79464 & 5.36849 & $\left(\mathrm{~d}_{2}, \mathrm{c}_{1}\right)$ & 1.41519 & 2.93378 & 5.74260 \\
\hline$\left(b_{3}, d_{0}\right)$ & 2.51944 & 4.93401 & 7.96091 & $\left(\mathrm{~d}_{2}, \mathrm{~d}_{0}\right)$ & 2.54455 & 5.32059 & 8.36534 \\
\hline$\left(b_{3}, d_{1}\right)$ & 1.56101 & 2.86121 & 5.38448 & $\left(\mathrm{~d}_{2}, \mathrm{~d}_{1}\right)$ & 1.68062 & 3.00844 & 5.75613 \\
\hline$\left(b_{3}, d_{2}\right)$ & 1.55171 & 2.90285 & 5.41903 & $\left(\mathrm{~d}_{2}, \mathrm{~d}_{2}\right)$ & 1.66921 & 3.05526 & 5.78994 \\
\hline
\end{tabular}

$\mathrm{BC}=$ boundary conditions as set out in Fig. 2, at points A and B, respectively (see Fig. 1).

System parameters: $\delta_{1}=0.1, \Delta_{1}=0.2, \varepsilon_{g, 1}=-0.1, \varepsilon_{p, 1}=-0.3, \delta_{2}=0.2, \Delta_{2}=0.3, \varepsilon_{g, 2}=0.3, \varepsilon_{p, 2}=0.2$. 
TABLE 4 The first three frequency parameters for a beam carrying heavy unrestrained end-bodies

\begin{tabular}{|c|c|c|c|c|c|c|c|c|c|}
\hline \multirow[b]{2}{*}{$\delta_{1}, \Delta_{1}$} & \multicolumn{3}{|c|}{$\varepsilon_{g, 1}=-0.1, \varepsilon_{g, 2}=-0.1$} & \multicolumn{3}{|c|}{$\varepsilon_{g, 1}=-0.1, \varepsilon_{g, 2}=0.0$} & \multicolumn{3}{|c|}{$\varepsilon_{g, 1}=-0.1, \varepsilon_{g, 2}=0.1$} \\
\hline & $\alpha_{1}$ & $\alpha_{2}$ & $\alpha_{3}$ & $\alpha_{1}$ & $\alpha_{2}$ & $\alpha_{3}$ & $\alpha_{1}$ & $\alpha_{2}$ & $\alpha_{3}$ \\
\hline 1,1 & 1.1833 & 2.2363 & 5.0656 & 1.1832 & 2.2551 & 5.0703 & 1.1814 & 2.2763 & 5.0774 \\
\hline $1,10^{2}$ & 0.3760 & 2.0975 & 5.0489 & 0.3760 & 2.0978 & 5.0490 & 0.3760 & 2.0980 & 5.0491 \\
\hline $1,10^{3}$ & uuu & 2.0961 & 5.0488 & uuu & 2.0962 & 5.0488 & uuu & 2.0962 & 5.0488 \\
\hline 10,1 & 1.1746 & 1.7081 & 4.7917 & 1.1798 & 1.7571 & 4.7981 & 1.1785 & 1.8120 & 4.8082 \\
\hline $10,10^{2}$ & 0.3760 & 1.2453 & 4.7699 & 0.3760 & 1.2467 & 4.7700 & 0.3760 & 1.2483 & 4.7701 \\
\hline $10,10^{3}$ & uuu & 1.2380 & 4.7697 & uuu & 1.2382 & 4.7698 & uuu & 1.2383 & 4.7698 \\
\hline $10^{2}, 1$ & 1.1696 & 1.5962 & 4.7569 & 1.1787 & 1.6555 & 4.7635 & 1.1781 & 1.7218 & 4.7740 \\
\hline $10^{2}, 10^{2}$ & 0.3752 & 0.7413 & 4.7343 & 0.3758 & 0.7477 & 4.7344 & 0.3760 & 0.7554 & 4.7345 \\
\hline $10^{2}, 10^{3}$ & uuu & 0.7040 & 4.7341 & uuu & 0.7048 & 4.7341 & uuu & 0.7057 & 4.7342 \\
\hline $10^{3}, 1$ & 1.1689 & 1.5834 & 4.7533 & 1.1786 & 1.6440 & 4.7599 & 1.1780 & 1.7117 & 4.7705 \\
\hline $10^{3}, 10^{2}$ & 0.3726 & 0.5432 & 4.7307 & 0.3753 & 0.5586 & 4.7307 & 0.3760 & 0.5764 & 4.7309 \\
\hline $10^{3}, 10^{3}$ & uuu & 0.4170 & 4.7305 & uuu & 0.4207 & 4.7305 & uuu & 0.4250 & 4.7305 \\
\hline $10^{4}, 1$ & 1.1688 & 1.5821 & 4.7529 & 1.1786 & 1.6428 & 4.7596 & 1.1780 & 1.7107 & 4.7701 \\
\hline $10^{4}, 10^{2}$ & 0.3710 & 0.5065 & 4.7303 & 0.3750 & 0.5247 & 4.7304 & 0.3760 & 0.5459 & 4.7305 \\
\hline $10^{4}, 10^{3}$ & uuu & uuu & 4.7301 & uuu & uuu & 4.7301 & uuu & 0.3242 & 4.7301 \\
\hline
\end{tabular}

иии, computations unsuccessful.

System parameters: $\varepsilon_{g, 1}$ and $\varepsilon_{g, 2}$ (in table) and $\delta_{1}=\delta_{2}$ and $\Delta_{1}=\Delta_{2}$.

$\Delta_{1}=\Delta_{2}$ was chosen. Pseudo-sliding-sliding boundary conditions are approached when $\delta_{1}=\delta_{2}=1, \Delta_{1}=\Delta_{2}=10^{3}$. The fundamental frequency is small and close to rigid-body translation. Computation of the fundamental frequency failed in double precession and is shown by иии in Table 4. Pseudo-clamped-clamped boundary conditions are approached when $\delta_{1}=\delta_{2}=10^{3}, \Delta_{1}=\Delta_{2}=10^{3}$. Here, the near-free body translation frequency is very small but the near-free body rotation is not very small. The combination $\delta_{1}=\delta_{2}=10^{4}, \Delta_{1}=\Delta_{2}=10^{3}$ is closer to a clamped-clamped condition and here the fundamental and second frequencies are very small but the third is very nearly equal to that of the classical clamped-clamped beam.

In Table 5, the first three frequency parameters of $\left(b_{3}, b_{3}\right)$ systems are tabulated for combinations of $\varepsilon_{p, 1}=-0.1,0.0$ or 0.1 and $\varepsilon_{p, 2}=-0.3,0.0$ or $0.3, \varepsilon_{g, 1}=-0.1$ and $\varepsilon_{g, 2}=0.2$ and selected combinations of $\delta_{1}=\delta_{2}=0$ or $10^{2}$ and $\Delta_{1}=\Delta_{2}=0,10^{-1}, 1$ or 10. The first three rows $\left(\delta_{1}=\delta_{2}=\Delta_{1}=\Delta_{2}=0\right)$, represent a uniform beam pinned at massless beam-like extensions and here the combination $\varepsilon_{p, 1}=0.0, \varepsilon_{p, 2}=0.0$ is the classical pinned-pinned beam. For large values of $\delta$ and/or $\Delta$, the system in the third mode asymptotically approaches the clamped-clamped condition. 
TABLE 5 The first three frequency parameters for a beam with pinned heavy rigid bodies at its ends

\begin{tabular}{|c|c|c|c|c|c|c|c|c|c|c|}
\hline \multirow{2}{*}{$\begin{array}{l}\left(\delta_{2}, \Delta_{2}\right) \\
\left(\delta_{1}, \Delta_{1}\right)\end{array}$} & \multirow[b]{2}{*}{$\varepsilon_{p, 1}$} & \multicolumn{3}{|c|}{$\varepsilon_{p, 2}=-0.3$} & \multicolumn{3}{|c|}{$\varepsilon_{p, 2}=0.0$} & \multicolumn{3}{|c|}{$\varepsilon_{p, 2}=0.3$} \\
\hline & & $\alpha_{1}$ & $\alpha_{2}$ & $\alpha_{3}$ & $\alpha_{1}$ & $\alpha_{2}$ & $\alpha_{3}$ & $\alpha_{1}$ & $\alpha_{2}$ & $\alpha_{3}$ \\
\hline \multirow[t]{3}{*}{$(0,0)$} & -0.1 & 3.3291 & 3.6209 & 6.8010 & 2.8628 & 5.7606 & 8.7083 & 2.3089 & 4.8610 & 7.6157 \\
\hline & 0.0 & 3.3247 & 4.0201 & 7.4324 & 3.1416 & 6.2832 & 9.4248 & 2.4984 & 5.2759 & 8.2385 \\
\hline & 0.1 & 3.3159 & 4.4972 & 8.1495 & 3.4761 & 6.8862 & 10.0000 & 2.7225 & 5.7596 & 8.9404 \\
\hline \multirow[t]{3}{*}{$\left(10^{2}, 10\right)$} & -0.1 & 0.4504 & 0.8540 & 4.7321 & 0.6291 & 0.9013 & 4.7327 & 0.6582 & 1.0308 & 4.7370 \\
\hline & 0.0 & 0.4526 & 0.7766 & 4.7309 & 0.6311 & 0.8368 & 4.7316 & 0.6422 & 0.9945 & 4.7358 \\
\hline & 0.1 & 0.4534 & 0.6758 & 4.7303 & 0.6116 & 0.7715 & 4.7309 & 0.5993 & 0.9640 & 4.7351 \\
\hline
\end{tabular}

†Classical pinned-pinned beam.

System parameters: $\varepsilon_{g, 1}=-0.1$ and $\varepsilon_{g, 2}=0.2, \varepsilon_{p, 1}$ and $\varepsilon_{p, 2}$ (in table) and $\delta_{1}=\delta_{2}$ and $\Delta_{1}=\Delta_{2}$.

\section{Example symmetrical systems}

Consider a beam carrying identical rigid bodies at its ends. The beam $A B$ is tubular, of length $L$, outside diameter $d_{0}=0.04 \mathrm{~m}$, inside diameter $d_{i}=0.03 \mathrm{~m}, E=205 \times$ $10^{9} \mathrm{~N} / \mathrm{m}^{2}$ and $\rho=7851.0 \mathrm{~kg} / \mathrm{m}^{3}$. The flexural rigidity is $E I=E \pi\left(\mathrm{d}_{0}^{4}-\mathrm{d}_{i}^{4}\right) / 64=$ $17610.1 \mathrm{Nm}^{2}$, the mass per unit length of the beam is $m=\rho \pi\left(\mathrm{d}_{0}^{2}-\mathrm{d}_{i}^{2}\right) / 4=4.316313$ $\mathrm{kg} / \mathrm{m}$. The cylindrical steel end-bodies (assumed rigid compared with the beam) are of diameter $D=0.3 \mathrm{~m}$ and axial width $w=0.10 \mathrm{~m}$. The mass is $M_{1}=M_{2}=M=$ $\rho w \pi D^{2} / 4=55.495449 \mathrm{~kg}$. The position of the centre of mass from the beam end is $\mathrm{e}_{\mathrm{g}, 1}=\mathrm{e}_{\mathrm{g}, 2}=w / 2=0.05 \mathrm{~m}$. The moment of inertia of the end-body about an axis through the centre of mass and normal to the pane of vibration is $J_{1}=J_{2}=J=$ $M\left(D^{2} / 16+w^{2} / 12\right)=0.358408 \mathrm{kgm}^{2}$. The mass parameter is $\delta_{1}=\delta_{2}=\delta=M / m L=$ $12.857143 / L$, the moment of inertia parameter is $\Delta_{1}=\Delta_{2}=\Delta=\mathrm{J} / \mathrm{mL}^{3}=0.0830357 / \mathrm{L}^{3}$ and the centre-of-mass offset parameter is $\varepsilon_{g, 1}=\varepsilon_{g, 1}=\varepsilon_{g}=w / 2 L$. From equation 2, a natural frequency is $\omega=\alpha^{2}[E I / m]^{1 / 2} / L^{2} \mathrm{rad} / \mathrm{s}$.

The beam carrying a rigid body at each end shown in Fig. 4(a) has boundary conditions $\left(\mathrm{d}_{2}, \mathrm{~d}_{2}\right)$. For a beam of length $L=2 \mathrm{~m}$, the system parameters are $\delta=6.42857$, $\Delta=0.01037946$ and $\varepsilon=0.025$. The first three frequency parameters (calculated from equation 18 with $U(1)$ and $V(1)$ obtained from equation 8) are $\alpha_{1}=2.79758, \alpha_{2}=$ 4.62953 and $\alpha_{3}=6.19687$ and the corresponding natural frequencies are $\omega_{1}=$ $124.977 \mathrm{rad} / \mathrm{s}, \omega_{2}=342.247 \mathrm{rad} / \mathrm{s}$ and $\omega_{3}=613.211 \mathrm{rad} / \mathrm{s}$. Because of symmetry, the mode shapes will be similar to those of a free-free uniform beam. There will be two nodes at the first non-zero frequency mode, three nodes at the second mode, and so on. For odd modes, the slope at the mid-point of the beam is zero; and for even modes, the mid-point is a node. The system shown in Fig. 4(b) is 'half' that shown in Fig. 4(a). Here the length of beam is $L=1 \mathrm{~m}$ and the system parameters are $\delta=$ $12.85714, \Delta=0.083033571$ and $\varepsilon=0.050$. The boundary conditions are $\left(\mathrm{c}_{0}, \mathrm{~d}_{2}\right)$. The first three frequency parameters (calculated from equation 18 with $U(1)$ and $V(1)$ 

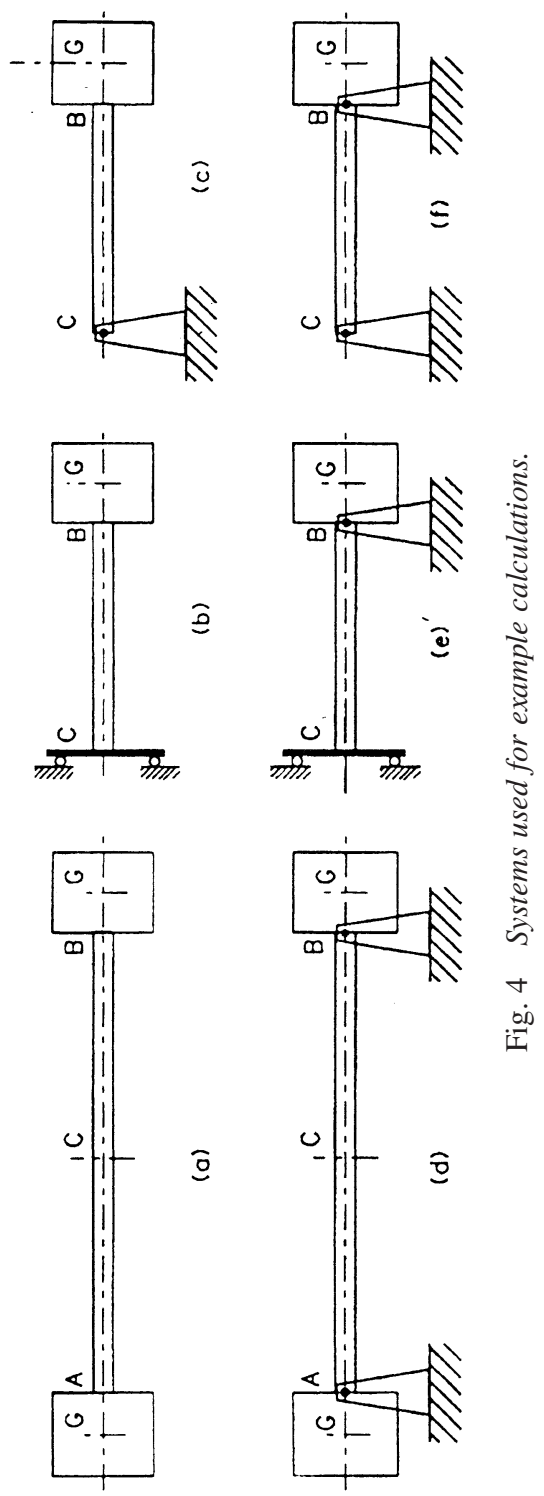
obtained from equation 12) are $\alpha_{1}=1.39879, \alpha_{2}=3.09844$ and $\alpha_{3}=5.62826$ and the corresponding natural frequencies are $\omega_{1}=124.977 \mathrm{rad} / \mathrm{s}, \omega_{2}=613.211 \mathrm{rad} / \mathrm{s}$ and $\omega_{3}=2023.359 \mathrm{rad} / \mathrm{s}$. Note that the odd mode frequencies of the system shown in Fig. 4(a) coincide with the frequencies of system shown in Fig. 4(b). The boundary conditions of the system shown in Fig. $4(c)$ are $\left(b_{0}, d_{2}\right)$. The system parameters are the same as those of Fig. 4(b). The first three frequency parameters are $\alpha_{1}=2.31477$, $\alpha_{2}=4.21379$ and $\alpha_{3}=7.14158$ and the corresponding natural frequencies are $\omega_{1}=$ $342.247 \mathrm{rad} / \mathrm{s}, \omega_{2}=1134.149 \mathrm{rad} / \mathrm{s}$ and $\omega_{3}=3257.711 \mathrm{rad} / \mathrm{s}$. Note that the even mode frequencies of the system shown in Fig. 4(a) coincide with the frequencies of system shown in Fig. 4(c).

The system shown in Fig. 4(d) is pinned at $A$ and at $B$; that is, the boundary conditions are $\left(b_{2}, b_{2}\right)$. The system parameters are the same as those in Fig. 4(a). The first three frequency parameters (calculated from equation 24 with $U(1)$ and $V(1)$ obtained from equation 15) are $\alpha_{1}=2.78492, \alpha_{2}=4.33466$ and $\alpha_{3}=5.76100$ and the corresponding natural frequencies are $\omega_{1}=123.848 \mathrm{rad} / \mathrm{s}, \omega_{2}=300.037 \mathrm{rad} / \mathrm{s}$ and $\omega_{3}=529.981 \mathrm{rad} / \mathrm{s}$. Because of symmetry, the frequency modes will be similar to those of a pinned-pinned uniform beam. The first mode shape will not have a node, the second mode will have one and so on. For odd modes, the slope at the mid-point of the beam is zero; for even modes, the mid-point is a node. The system shown in Fig. 4(e) and 4(f) are 'half' that in Fig. 4(d). The boundary conditions of the system in Fig. $4(\mathrm{e})$ is $\left(\mathrm{c}_{0}, \mathrm{~b}_{2}\right)$ and the first three frequency parameters are $\alpha_{1}=1.39246, \alpha_{2}$ $=2.88050$ and $\alpha_{3}=5.55122$ and the corresponding natural frequencies are $\omega_{1}=$ $123.848 \mathrm{rad} / \mathrm{s}, \omega_{2}=529.981 \mathrm{rad} / \mathrm{s}$ and $\omega_{3}=1968.346 \mathrm{rad} / \mathrm{s}$. The odd mode frequencies of the system shown in Fig. 4(d) coincide with the frequencies of system shown in Fig. 4(e). The boundary conditions of the system in Fig. 4(f) is $\left(b_{0}, b_{2}\right)$ and the first three frequency parameters are $\alpha_{1}=2.16733, \alpha_{2}=4.07300$ and $\alpha_{3}=7.09351$ and the corresponding natural frequencies are $\omega_{1}=300.037 \mathrm{rad} / \mathrm{s}, \omega_{2}=1059.629$ $\mathrm{rad} / \mathrm{s}$ and $\omega_{3}=3214.006 \mathrm{rad} / \mathrm{s}$. The even mode frequencies of they system shown in Fig. 4(d) coincide with the frequencies of system shown in Fig. 4(f).

\section{Concluding remarks}

The vibration of a Euler-Bernoulli uniform beam carrying a rigid body at each end was considered in this paper. Combinations of boundary conditions were considered: classical clamped, pinned, sliding or free, the translation and rotation of an unrestrained end-body, the translation and/or rotation of a restrained end-body and special boundary conditions. The frequency equations were expressed as a secondorder determinant equated to zero. Schemes were presented to obtain the elements of the determinant and to calculate the natural frequency parameters. The first three frequency parameters of a beam with a body attached at its ends and 'free' to translate and rotate were tabulated. The next case considered was where the end-body is pinned. Special and degenerate combinations of boundary conditions were classified into 100 types. The first three frequency parameters were tabulated for selected sets of system parameters. Combinations of classical 'clamped', 'pinned', 'sliding' or 'free' boundary conditions were not included. 
The trend in frequency changes when one or more system parameters are increased indefinitely, as demonstrated in the tables, and this was explained from physical considerations. Computations in double precision were not successful for combinations of system parameters for which the natural frequency parameter is small. Computations would be successful with higher-precision arithmetic. The results presented (for several conceivable systems) may be used to judge frequencies obtained by numerical methods.

\section{References}

[1] H. McCallion, Vibration of Linear Mechanical Systems (Longman, London, 1973).

[2] D. J. Gorman, Free Vibration Analysis of Beams and Shafts (Wiley, London, 1975).

[3] A. H. Register, 'A note on the vibrations of generally restrained, end loaded beams', Journal of Sound and Vibration, 172(4) (1994), 561-571.

[4] B. Rama Bhat and M. A. Kulkarni, 'Natural frequencies of a cantilever with slender tip mass', AIAA Journal, 14(4) (1976), 536-537.

[5] B. Rama Bhat and H. Wagner, 'Natural frequencies of a cantilever with a tip mass slender in the axial direction', Journal of Sound and Vibration, 45(2) (1976), 304-307.

[6] N. Popplewell and D. Chang, 'Free vibrations of complex Euler-Bernoulli beam', Journal of Sound and Vibration, 190(5) (1996), 852-856. 